\title{
CORRECTIONS
}

\section{Andrew Witty: the acceptable face of big pharma?}

In this Feature we mistakenly spelt out the FDA as "the Food and Drink Administration," instead of the Food and Drug

Cite this as: BMJ 2013;346:f1661

Administration (BMJ 2013;346:f1458, doi:10.1136/bmj.f1458).

๑ BMJ Publishing Group Ltd 2013 